\title{
A pharmacodynamic evaluation of midazolam as an antiepileptic compound
}

\author{
S JAWAD,* J OXLEY, $\dagger$ J WILSON,* A RICHENS* \\ From the Department of Pharmacology and Therapeutics, University Hospital of Wales, ${ }^{*}$ Cardiff and the \\ National Society for Epilepsy, Chalfont Centre for Epilepsy, Chalfont St Peter, $\dagger$ UK
}

SUMMARY Midazolam is a water soluble 1,4 benzodiazepine which is suitable for intramuscular administration. It is currently used for pre-medication and the induction of anaesthesia. Its antiepileptic properties have been evaluated by studying its effect on interictal spikes on the EEG of six adult epileptic patients. The results indicate that intramuscular midazolam $15 \mathrm{mg}$ is more effective than intramuscular diazepam $10 \mathrm{mg}$ in abolishing interictal spikes and as effective as intravenous diazepam $20 \mathrm{mg}$ five minutes after administration.

Midazolam is a new 1,4 benzodiazepine drug belonging to the group of 1,2 annelated benzodiazepines, which have in common a five membered heterocyclic ring fused on position 1,2 of the diazepine nucleus, and have a high affinity to benzodiazepine receptors. ${ }^{1}$ It is unique in the basicity of its molecule which permits the preparation of water soluble salts, giving a very stable aqueous injectable solution. It has a short duration of action due to rapid metabolic inactivation, and has been used successfully for the intravenous induction of anaesthesia. ${ }^{2}$ Animal data ${ }^{3}$ have suggested that midazolam might be useful as an intravenous or intramuscular antiepileptic drug.

Ahmad et al $^{4}$ reported that EEG spike counting could be used for the rapid assessment of new antiepileptic compounds given in single intravenous doses. Milligan et $a^{56}$ modified the technique and successfully demonstrated the efficacy of rectal diazepam and intravenous phenytoin in suppressing interictal spikes. We have used this method to assess the comparative efficacy of midazolam and diazepam.

\section{Methods}

Six adult epileptic patients (four male, two female, age range 32-57 years), resident at the Chalfont Centre for Epilepsy, gave their signed consent to take part in this study. Their routine drug therapy, which consisted of carbamazepine (one subject), carbamazepine and primidone (one subject), carbamazepine and phenytoin (two subjects), car-

Address for reprint requests: Dr J Oxley, Chalfont Centre for Epilepsy, Chalfont St Peter, Gerrards Cross, Bucks SL9 0RJ, UK.

Received 28 June 1985 and in revised form 20 November 1985. Accepted 23 November 1985 bamazepine and sodium valproate (one subject) and phenytoin and sodium valproate (one subject), was kept unchanged throughout. None suffered from severe seizures requiring the use of benzodiazepines for at least three weeks before and during the study. The subjects were selected on the basis of having frequent spontaneous interictal spikes, seen as isolated spikes, polyspikes and spike and wave discharges, in their routine EEGs. All the intramuscular injections were given rapidly in the upper outer quadrant of the right buttock, while the intravenous injections were given at a rate of $0.5 \mathrm{ml} / \mathrm{min}$ into an antecubital vein by one experimenter (SJ).

The comparative evaluation of midazolam and diazepam was carried out in three parts.

\section{Part 1}

In this study the effects of intramuscular midazolam $10 \mathrm{mg}$ and $15 \mathrm{mg}$, intravenous diazepam $10 \mathrm{mg}$ and $20 \mathrm{mg}$ and placebo were compared. Each patient received randomly the following treatments separated by intervals of 10 days:

$\mathrm{T} 1$ : intravenous normal saline + intramuscular normal saline

T2: intravenous normal saline + intramuscular midazolam $15 \mathrm{mg}$

T3: intravenous normal saline + intramuscular midazolam $10 \mathrm{mg}$

T4: intravenous diazepam $10 \mathrm{mg}+$ intramuscular normal saline

T5: intravenous diazepam $20 \mathrm{mg}+$ intramuscular normal saline

A 10 minute baseline EEG was recorded with the subjects' eyes closed immediately before drug administration and this was used as a control. Five minutes after drug administration, nine subsequent 10 minute recordings were made every 20 minutes over a 3 hour period. EEG recordings were achieved using an eight channel SLE (Galileo Model E88) electroencephalograph recorder and bipolar electrodes positioned according to the 10-20 system. All the recordings 
were made from the lateral ring of electrodes. The EEGs were then code numbered and subsequently analysed blind and in random order using the method described by Ahmad et al. ${ }^{4} \mathrm{~A}$ spike was defined as a waveform distinguished from background activity and having a duration less than $70 \mathrm{~ms}$ (Cooper et al). ${ }^{7}$ Sharp waves were included in the counting where these were clearly of epileptic origin, that is accompanied by spikes or sharp waves in adjacent channels or where they were phase reversing. In general, the duration of sharp waves included did not exceed $150 \mathrm{~ms}$ (accepted range $70-200 \mathrm{~ms}$ ). Spike counting was confined to the one EEG channel in which the spikes were most clearly defined. The total number of spikes in each 10 minute recording was compared for different treatments using a computerised threeway analysis of variance.

\section{Part 2}

The same EEG assessment and analysis procedures were used in the same six subjects to compare directly the effects of intramuscular midazolam $15 \mathrm{mg}$ (T6) and intramuscular diazepam $10 \mathrm{mg}$ (T7) over a 3 hour period. No placebo was used as the effectiveness of intramuscular midazolam against placebo had already been demonstrated in Part 1 .

\section{Part 3}

As the timing of the first EEG recording was set at 5 minutes after injection this did not allow adequate differentiation of the speed of onset of action between the intravenous and intramuscular preparations. As it was anticipated that a benzodiazepine which could be given intramuscularly might be of use in the emergency treatment of seizures, the immediate effects of intramuscular midazolam $15 \mathrm{mg}$ and intravenous normal saline (T8) and intramuscular normal saline and intravenous diazepam $20 \mathrm{mg}$ (T9) were compared in the same six subjects. The EEG was recorded for 10 minutes prior to and after parenteral injection. The EEGs were coded and analysed later in a blind fashion. The number of spikes in each minute was counted and the results analysed by computerised analysis of variance.

\section{Results}

\section{Part I}

All the patients fell asleep 11/2-3 minutes following midazolam $15 \mathrm{mg}$ and diazepam $20 \mathrm{mg}, 2-4$ minutes following midazolam $10 \mathrm{mg}$ and 3-4 minutes following diazepam $10 \mathrm{mg}$. All the subjects fell asleep 30-45 minutes following placebo. All the patients were left undisturbed and woke up spontaneously at the end of the recording. No adverse effects were noted during the period of the study, apart from amnesia produced by midazolam.

The mean spike counts for different treatments at different times are given in table 1 and illustrated graphically in the figure.

All the main effects, that is difference between patients, time and treatments, were highly significant on an analysis of variance $(p<0.01)$.

In addition, the two-way interactions were all highly significant, that is the patients had significantly different time courses for their drug effects and significantly different degrees of response. There were also significant differences between the time courses of the effects of the treatments. Since the latter was significant, the main effects were re-tested using the pooled residual and two-way interaction sums of squares to make sure that the main effects were not due to the two-way interactions. Again, all main effects were still highly significant. Thus, there was a highly significant difference between drug treatments. An estimate of the standard error of a treatment mean at each time point was $7 \cdot 353$.

In order to detect which treatments were significantly different, two range tests on the five treatment means were carried out. The first was the least significant difference test at $p<0.05$, and the

Table 1 Mean number of spikes $[ \pm S D]$ in each 10 min $E E G$ recording following treatments $T 1-T 7$

\begin{tabular}{|c|c|c|c|c|c|c|c|}
\hline Time (min) & $T I$ & $T 2$ & $T 3$ & $T 4$ & $T 5$ & $T 6$ & $T 7$ \\
\hline $\begin{array}{c}-10-0 \\
\text { [baseline] } \\
5-15 \\
25-35 \\
45-55 \\
65-75 \\
85-95 \\
105-115 \\
125-135 \\
145-155 \\
165-175\end{array}$ & $\begin{array}{l}81.8 \pm 23.6 \\
78.5 \pm 22.0 \\
93.0 \pm 15.8 \\
78.3 \pm 22.5 \\
59.7 \pm 15.8 \\
64.2 \pm 16.3 \\
55.8 \pm 12.3 \\
53.2 \pm 11.3 \\
66.3 \pm 20.7 \\
86.5 \pm 8.8\end{array}$ & $\begin{array}{c}83.5 \pm 21.3 \\
2.0 \pm 3.6 \\
5.3 \pm 6.0 \\
12.5 \pm 12.9 \\
5.3 \pm 5.0 \\
7.7 \pm 5.5 \\
13.0 \pm 14.2 \\
19.8 \pm 26.5 \\
20.3 \pm 25.7 \\
20.0 \pm 24.3\end{array}$ & $\begin{array}{l}77.0 \pm 29.2 \\
8.2 \pm 9.4 \\
12.2 \pm 16.5 \\
15.5 \pm 29.5 \\
17.2 \pm 24.7 \\
16.7 \pm 25.4 \\
16.2 \pm 20.6 \\
16.5 \pm 18.1 \\
20.5 \pm 18.2 \\
19.2 \pm 17.3\end{array}$ & $\begin{array}{l}78.3 \pm 24.7 \\
16.5 \pm 14.8 \\
10.5 \pm 7.9 \\
11.7 \pm 14.0 \\
13.2 \pm 16.7 \\
26.8 \pm 25.8 \\
31.8 \pm 31.0 \\
37.8 \pm 32.3 \\
39.3 \pm 37.7 \\
39.2 \pm 35.2\end{array}$ & $\begin{array}{c}70.8 \pm 13.1 \\
2.8 \pm 2.9 \\
3.0 \pm 3.2 \\
6.7 \pm 7.9 \\
6.5 \pm 7.5 \\
8.7 \pm 7.1 \\
13.0 \pm 17.5 \\
12.2 \pm 14.4 \\
17.3 \pm 12.8 \\
17.2 \pm 11.0\end{array}$ & $\begin{array}{r}136.5 \pm 68.8 \\
18.3 \pm 18.2 \\
16.5 \pm 20.0 \\
8.0 \pm 9.0 \\
9.8 \pm 11.6 \\
13.7 \pm 14.8 \\
12.2 \pm 13.7 \\
12.2 \pm 17.0 \\
16.3 \pm 18.3 \\
15.2 \pm 16.7\end{array}$ & $\begin{array}{r}130.8 \pm 61.1 \\
75.0 \pm 66.3 \\
72.8 \pm 64.6 \\
54.2 \pm 50.5 \\
63.7 \pm 54.3 \\
70.0 \pm 61.7 \\
71.8 \pm 61.2 \\
76.7 \pm 68.3 \\
63.2 \pm 52.0 \\
64.8 \pm 53.6\end{array}$ \\
\hline
\end{tabular}

T1: intravenous normal saline + intramuscular normal saline.

T2: intravenous normal saline + intramuscular midazolam $15 \mathrm{mg}$.

T3: intravenous normal saline + intramuscular midazolam $10 \mathrm{mg}$.

T4: intravenous diazepam $10 \mathrm{mg}$ + intramuscular normal saline.

T5: intravenous diazepam $20 \mathrm{mg}$ + intramuscular normal saline.

T6: intramuscular midazolam $15 \mathrm{mg}$.

T7: intramuscular diazepam $10 \mathrm{mg}$. 


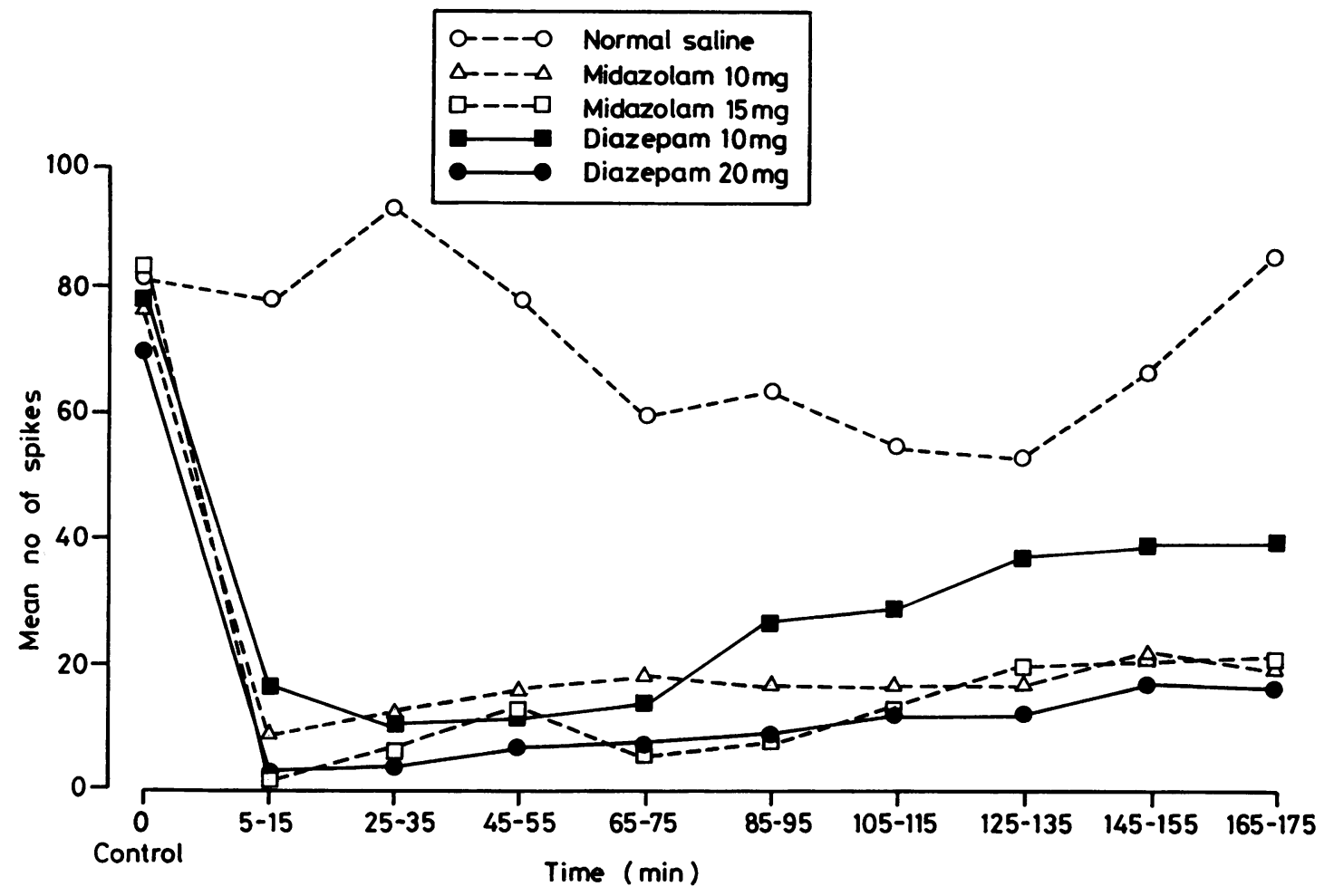

Fig Mean number of spikes versus time following different treatments.

second the Student Newman-Keuls test at $p<0.05$, which avoids certain problems of over sensitivity in the least significant difference test. In this case, however, both tests produced the same result. Treatment 1 (placebo) was significantly different from all other treatments. Treatment 4 (intramuscular normal saline and intravenous diazepam $10 \mathrm{mg}$ ) was also significantly different from all other treatments. All the other treatments, that is treatment 2 (intravenous normal saline and intramuscular midazolam $15 \mathrm{mg}$ ), treatment 3 (intravenous normal saline and intramuscular midazolam $10 \mathrm{mg}$ ) and treatment 5 (intramuscular normal saline and intravenous diazepam $20 \mathrm{mg}$ ) did not differ.

\section{Part 2}

All the subjects slept following intramuscular midazolam. None of the subjects slept following intramuscular diazepam and all of them complained of moderate pain at the site of injection.

Table 1 shows the mean number of spikes following intramuscular midazolam $15 \mathrm{mg}$ (T6) versus intramuscular diazepam $10 \mathrm{mg}$ (T7). The analysis of variance showed significant interaction terms for both subject-drug and drug-time. The main effects were retested against the combined residual and interaction variances, and " $F$ value" for the differences between drugs was still highly significant $p<0.001$. This indicated the superiority of intramuscular midazolam $15 \mathrm{mg}$ over intramuscular diazepam $10 \mathrm{mg}$ in suppressing interictal spikes.

\section{Part 3}

Table 2 gives the mean number of spikes at each minute following intramuscular midazolam $15 \mathrm{mg}+$ intravenous normal saline (T8) and intravenous diazepam $20 \mathrm{mg}+$ intramuscular normal saline (T9). A quantitative analysis of the difference in rate of onset of drug action proved difficult from the data obtained. It involved fitting the data to some empirical model. A simple approach was adopted, assuming that the drug effect builds exponentially, so a plot of log response against time might be approximately linear up to the attainment of maximum response. The mean data were treated in this way because of presence of zeros in the individual patient data. The scatter of the diazepam data followed a log linear fit, but for the midazolam data a sigmoid curve 
Table 2 Mean number of spikes [ $\pm S D$ ] per minute following treatments $T 8-T 9$

\begin{tabular}{|c|c|c|}
\hline Time (min) & $T 8$ & $T 9$ \\
\hline $\begin{array}{l}\text { Baseline } \\
0-1 \\
1-2 \\
2-3 \\
3-4 \\
4-5 \\
5-6 \\
6-7 \\
7-8 \\
8-9 \\
9-10\end{array}$ & $\begin{array}{r}13.8 \pm 5.9 \\
12.8 \pm 4.8 \\
12.3 \pm 3.7 \\
9.8 \pm 6.6 \\
5.8 \pm 7.5 \\
3.2 \pm 3.1 \\
2.7 \pm 4.2 \\
3.8 \pm 5.5 \\
3.7 \pm 4.8 \\
3.5 \pm 5.0 \\
3.3 \pm 4.5\end{array}$ & $\begin{array}{r}12.5 \pm 4.9 \\
6.2 \pm 6.2 \\
5.5 \pm 5.5 \\
4.2 \pm 4.7 \\
4.7 \pm 4.7 \\
3.0 \pm 2.8 \\
1.5 \pm 2.1 \\
0.7 \pm 1.2 \\
1.0 \pm 1.5 \\
1.2 \pm 1.9 \\
1.3 \pm 2.3\end{array}$ \\
\hline
\end{tabular}

T8: intramuscular midazolam $15 \mathrm{mg}+$ intravenous normal saline. T9: intravenous diazepam $20 \mathrm{mg}+$ intramuscular normal saline.

was obtained, suggesting that the model is not entirely suitable and that delay of 2-3 min may occur before the response builds up at a rate similar to that of diazepam. These conclusions are qualitative and do not permit more complex numerical analysis.

\section{Discussion}

The assessment in humans of new compounds with antiepileptic potential has been dependent traditionally on clinical trials. However, attempts have been made recently to devise techniques which permit new drugs to be evaluated at an earlier stage ${ }^{8}$ but only when a large number of compounds have been assessed will it be possible to validate these methods as predictors of antiepileptic efficacy in patients. The technique of interictal spike counting, following a single IV or oral dose, has so far been used to examine proven antiepileptic drugs, namely rectal diazepam, ${ }^{5}$ phenytoin, ${ }^{6}$ and an experimental compound, lamotrigine, ${ }^{9}$ which is currently undergoing clinical trials and frusemide. Interestingly, intravenous frusemide ${ }^{4}$ which is not generally regarded as an antiepileptic drug significantly reduced interictal spikes and was subsequently shown to be an effective antiepileptic agent when given as add-on therapy. ${ }^{10}$ Sodium valproate, on the other hand, when given intravenously at a dose of $15 \mathrm{mg} / \mathrm{kg}$ had no effect on interictal spikes (Milligan, unpublished). This latter finding may reflect a different mode of action of valproate in suppressing seizure activity.

The results of this study show that intramuscular midazolam at doses of $10 \mathrm{mg}$ and $15 \mathrm{mg}$ produced a significant pharmacodynamic effect as measured by a reduction in spike counts in the EEG. This effect was comparable at 5 minutes after injection to that produced by intravenous diazepam $20 \mathrm{mg}$. Intramuscular midazolam $15 \mathrm{mg}$ was, however, more effective than diazepam $10 \mathrm{mg}$ given intramuscularly or intravenously.
It is highly unlikely that suppression of interictal spikes is only a non-specific effect due to sedation. In Part 1 of the study all subjects fell asleep after each treatment and although a non significant fall in spikes was seen after placebo the mean spike counts were still significantly lower after all active treatments. In Part 2 of the study subjects remained awake for 45 minutes after diazepam $10 \mathrm{mg}$ intramuscularly but nevertheless a significant fall in spike counts was observed.

Although in the first part of this study intramuscular midazolam and intravenous diazepam appeared to be equally effective in abolishing interictal spikes, it should be noted that the first measurement was not made until 5 minutes after injection, and it is possible that intravenous diazepam produced a greater effect within this time. Similarly, the nature of the assessment of drug action, that is by counting EEG spikes, was unsuitable for determining the speed of onset of action of the intravenously administered drug. However, a qualitative assessment of the data obtained would indicate a delay in the onset of action of midazolam compared with diazepam of 2-3 minutes.

A drug which can be given intramuscularly and which effectively and rapidly suppresses interictal spikes may be of considerable clinical value in the emergency treatment of seizures. Although intravenous diazepam is generally regarded in the UK as the treatment of choice in this situation, it may be that intramuscular midazolam will have a comparable effect in suppressing seizures and the anticipated delay in onset of action of 2-3 minutes may well be acceptable in clinical situations where it is not possible for an intravenous injection to be given. The results of this study and preliminary clinical experience $^{11}$ indicate that intramuscular midazolam should be evaluated further in a controlled trial against intravenous diazepam.

Diazepam solution and midazolam solution were kindly supplied by Roche Products Ltd. Our thanks are due to Mrs Margaret Roberts for assistance with EEG recordings, Mrs Joanna Rope for preparing the typescript and the people with epilepsy who agreed to take part in this study.

\section{References}

${ }^{1}$ Gerecke M. Chemical structure and properties of midazolam compared with other benzodiazepines. $\mathrm{Br} \mathrm{J} \mathrm{Clin}$ Pharmacol 1983;16:11S-16S.

${ }^{2}$ Conner JT, Katz RL, Pagano RP, Graham CW. PO21-3981 for intravenous surgical pre-medication and induction of anaesthesia. Anesthiol Analg 1978;57:1-5. 
${ }^{3}$ Pieri L. Preclinical pharmacology of midazolam. Br J Clin Pharmacol 1983;16:17S-27S.

${ }^{4}$ Ahmad S, Perucca E, Richens A. The effect of frusemide, mexilitine, $(+)$ propranolol and three benzodiazepine drugs on inter-ictal spike discharges in the electroencephalogram of epileptic patients. Br J Clin Pharmacol 1977;4:683-8.

${ }^{5}$ Milligan N, Dhillon S, Oxley J, Richens A. Absorption of diazepam from the rectum and its effect on inter-ictal spikes in the EEG. Epilepsia 1982;23:323-31.

${ }^{6}$ Milligan N, Oxley J, Richens A. Acute effects of intravenous phenytoin on the frequency of inter-ictal spikes in man. Br J Clin Pharmacol 1983;16:285-9.
${ }^{7}$ Cooper R, Osselton JW, Shaw JC. EEG Technology. London: Butterworths, 1980:131.

${ }^{8}$ Milligan N, Richens A. Methods of assessment of antiepileptic drugs. Br J Clin Pharmacol 1981;11:443-56.

${ }^{9}$ Jawad S, Oxley JR, Yuen WC Richens A. Reduction of interictal electroencephalographic spikes by lamotrigine in epileptic patients. $\mathrm{Br} J$ Clin Pharmacol 1985;20:287.

${ }^{10}$ Ahmed S, Clark L, Hewitt AJ, Richens A. Controlled trial of frusemide as an antiepileptic drug in focal epilepsy. Br J Clin Pharmacol 1976;3:621.

11 Jawad S, Richens A, Oxley J. Pharmacodynamic and clinical evaluation of midazolam in epilepsy. Acta Neurol Scand 1984;70:219. 